\title{
Size and Concentration Analysis of Gold Nanoparticles With Ultraviolet-Visible Spectroscopy
}

Sanim Rahman

University of South Florida

\author{
Advisors: \\ Jonathan Burns, Mathematics and Statistics \\ Venkat Bhethanabotla, Chemical and Biomedical Engineering \\ Parveen Bazard, Global Center for Hearing and Speech Research \\ Problem Suggested By: Parveen Bazard
}

Follow this and additional works at: https://digitalcommons.usf.edu/ujmm

Part of the Mathematics Commons

UJMM is an open access journal, free to authors and readers, and relies on your support:

Donate Now

\section{Recommended Citation}

Rahman, Sanim (2016) "Size and Concentration Analysis of Gold Nanoparticles With Ultraviolet-Visible Spectroscopy," Undergraduate Journal of Mathematical Modeling: One + Two: Vol. 7: Iss. 1, Article 2. DOI: http://dx.doi.org/10.5038/2326-3652.7.1.4872

Available at: https://digitalcommons.usf.edu/ujmm/vol7/iss1/2 


\title{
Size and Concentration Analysis of Gold Nanoparticles With Ultraviolet-Visible Spectroscopy
}

\author{
Abstract \\ Spherical gold nanoparticles (GNPS) are synthesized by Turkevich Method and PEL 35 UV-VIS \\ spectrophotometer recorded the wavelength and absorption of the Surface Plasmon Resonance (SPR) \\ peak. The diameter and concentration of solute GNPs are calculated. The concentration of GNPs is done \\ with Beer's law. The average diameter of GNPs is done via the ratio of SPR peak to the absorbance at 450 \\ $\mathrm{nm}$. The diameters are compared to SEM scan of synthesized GNPs and Sigma-Aldrich values.
}

\section{Keywords}

gold nanoparticles, Beer-Lambert Law, Mie Theory

Creative Commons License

(c) (i) (9)

This work is licensed under a Creative Commons Attribution-Noncommercial-Share Alike 4.0 License. 


\section{PROBLEM STATEMENT}

The Turkevich Method, one of the most widely accepted protocols for gold nanoparticle (GNP) synthesis, produces polydisperse gold nanoparticles (Turkevich, Stevenson and Hillier). Due to the range of sizes, it may be difficult to determine the average size of these synthesized nanoparticles. Although there are set protocols to obtain a certain average size, the synthesis is extremely sensitive which makes a characterization of the produced nanoparticles a necessity. Another issue is that the theoretical concentration of gold nanoparticles suspended in solution may not be accurately depicted depending on whether the reaction is fully complete.

A possible method to characterize these gold nanoparticles is by observing their lightscattering properties. According to the Mie Theory, different-sized gold nanoparticles exhibit unique light-scattering properties (Mie). By observing these trends by collecting their absorption at their Surface Plasmon Resonance (SPR) and at a wavelength of $450 \mathrm{~nm}$, the average size of the gold nanoparticles can be estimated. With Beer-Lambert's law, the concentration of gold nanoparticles in solution was calculated.

\section{MOTIVATION}

With the emergence of nanomedicine in the past decades, the utilization of GNPs has been recognized due to its optical, electrochemical, and physical properties. Particularly, GNPs within the size range of 1-100 nanometers feature significant promise in biotechnology with applications in cellular imaging, cochlear implants, drug delivery etc. However, each size nanoparticle presents its own unique set of properties, therefore, making it crucial to know the size of the GNPs. More complications can arise when producing GNPs by the Turkevich method. 
Through this method it is nearly impossible to create exact monodispersed nanoparticles, therefore, a variety of different size will exist in the solution.

The most common forms of determining the size of GNPs is through utilization of a Transmission Electron Microscopy (TEM), Scanning Electron Microscopy (SEM), or Dynamic Light Scattering (DLS) Instrument. However, these instruments may be out of reach or unaffordable to use. A UV-VIS Spectroscopy can be an alternative for any of the instruments to obtain data about the produced GNPs. Through the application of Calculus, one can make low error estimates about the concentration and average size of the GNPs produced.

\section{MATHEMATICAL DESCRIPTION AND SOLUTION APPROACH}

Using the data collected through the UV-Vis Spectrophotometer, determine the wavelength and absorption where the SPR peak occurs.

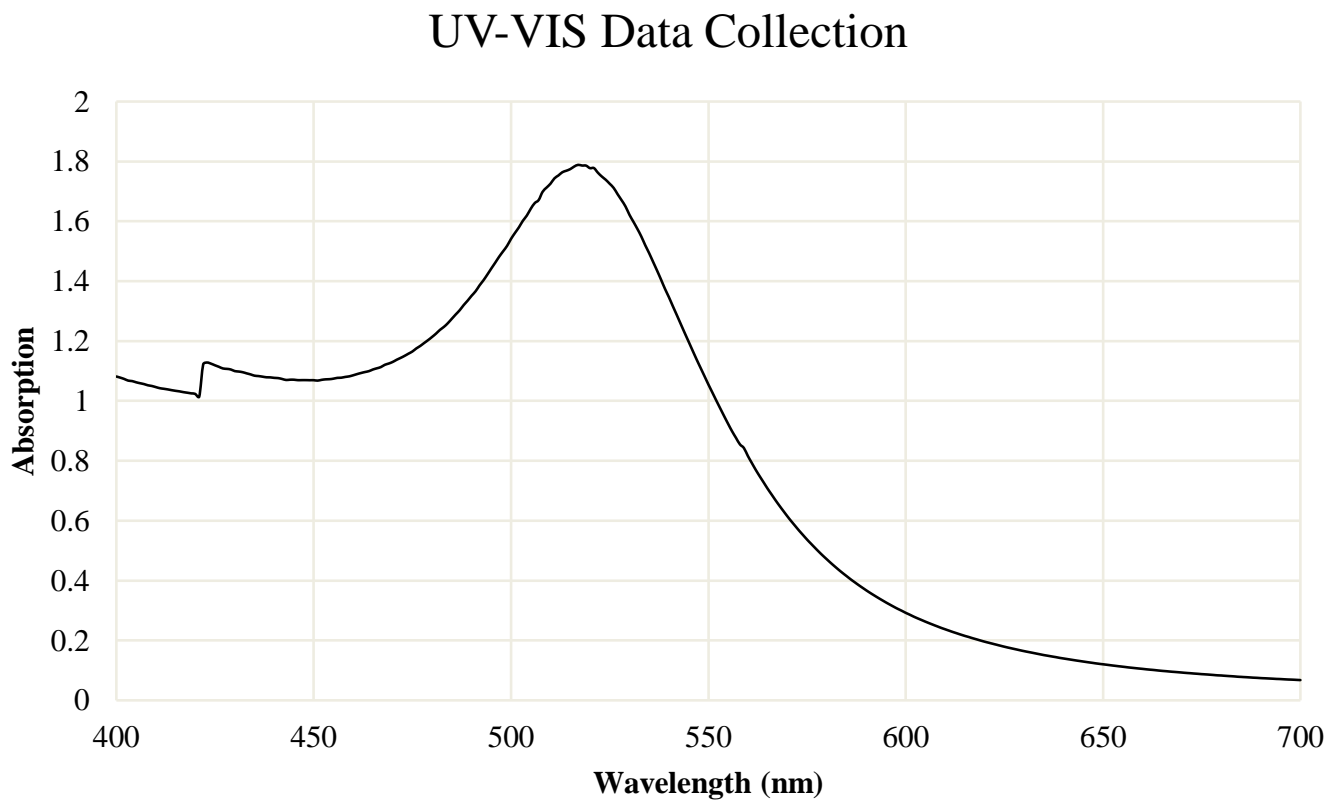


Figure 1: Above represents a UV-VIS analysis of a GNP sample from the Perkin Elmer Lambda 35 UV/Vis spectrophotometer. The absorption has been recorded from a wavelength of 400 to 700 . SPR

$$
\text { Peaks occurs at }(517,1.788574)
$$

In order to calculate the concentration of gold nanoparticles in solution, refer to Beer Lambert's Law (Swineheart, D. F.).

$$
A=\epsilon c f
$$

Where the product of molar concentration $(c)$, molar absorptivity $(\epsilon)$, and the path length $(b)$ equals to absorbance.

Through the Turkevich method involving the manipulations provided by Dr. Kumar (Kumar, Dhiraj; Meenan J., Brian; Mutreja, Isha; D'sa, Raechelle; Dixon Dorian), two attenuating species will exist in the solution (trisodium citrate dihydrate and gold (III) chloride trihydrate). Therefore, the absorbance of the GNP $\left(A_{G N P}\right)$ would be represented as:

$$
A_{G N P}=A_{1}+A_{2}=\epsilon_{1} \mathrm{c}_{1} \mathrm{f}+\epsilon_{2} \mathrm{c}_{2} \mathrm{f}
$$

Where the sum of the absorbance of trisodium citrate dihydrate $\left(A_{l}\right)$ and the absorbance of gold (III) chloride trihydrate $\left(A_{2}\right)$ in the solution is equal to the absorbance of the GNPs. Then solve for the concentration of gold (III) trihydrate in the solution.

Solve for the amount of moles gold in the solution and then multiply by Avogrado's number to determine how many particles were produced in the synthesis.

The amount of atoms to form a nanoparticle $(N)$ with diameter $(d)$ would be defined as (Lu, Yan; Wang, Lixia; Chen, Dejun; Wang, Gongke):

$$
N=\frac{\pi \rho d^{3}}{6 M}
$$

Where one must consider both density of gold $(\rho)$, molar mass of gold $(M)$, and the diameter in nanometers $(d)$. 
By knowing how many gold atoms consist of a single GNP, one can determine how many GNPs are formed in the solution by taking the quotient of GNPs in solution by number gold atoms to produce a single atom.

One then can calculate the concentration of the solution by dividing the number of nanoparticles by the volume of the solution.

Another approach to determining the concentration of GNs in solution would to assume that the entire reducing agent has been used up and only one attenuating species exist in solution, the GNPs. The absorbance of the GNPs would be represented as:

$$
A_{G N P}=\epsilon_{G N P} c_{G N P} f
$$

Where $\epsilon_{\mathrm{GNP}}$ would be represented as the molar absorptivity of the GNPs and $c_{G N P}$ represents the concentration of the GNPs.

Referring to Figure 1, use the absorption at the SPR for the absorption of the GNPs to solve for the concentration of gold nanoparticles. The extinction coefficient for GNPs have an SPR peak at a wavelength of 515-520 was obtained from Sigma-Aldrich (Appendix, Table [2]). To determine the path length $(f)$, the path length of the cuvette was used in the Perkin Elmer Lambda 35 UV/Vis spectrophotometer.

Using Equation (4), solve for the concentration of GNPs in the solution. With the molarity and volume of the sample, the moles of the GNPs can be calculated. With the moles of GNPs, multiply it by Avogadro's number to get the total number of GNPs in the solution. With the total number of GNPs in solution, find the concentration by dividing it by the volume of solution.

In order to determine the average diameter, the diameter of a GNP $(d)$ can be represented as (Haiss, Wolfgang; Nguyen T. K., Thanh; Aveyard, Jenny; Fernig G., David): 


$$
d=e^{\left(B_{1} \frac{A_{\text {spr }}}{A_{450}}-B_{2}\right)}
$$

By knowing the absorption at the SPR peak and $450 \mathrm{~nm}$, an estimate of the GNPS can be determined if the particles are between 5 to $80 \mathrm{~nm}$. Variables $\mathrm{B}_{1}$ and $\mathrm{B}_{2}$ are calculated values based on one's own produced data. $\mathrm{B}_{1}$ is represented as the inverse of the slope of the plotted values in Figure 2 and $\mathrm{B}_{2}$ is represented as the $\mathrm{y}$-axis intercept divided by the slope.

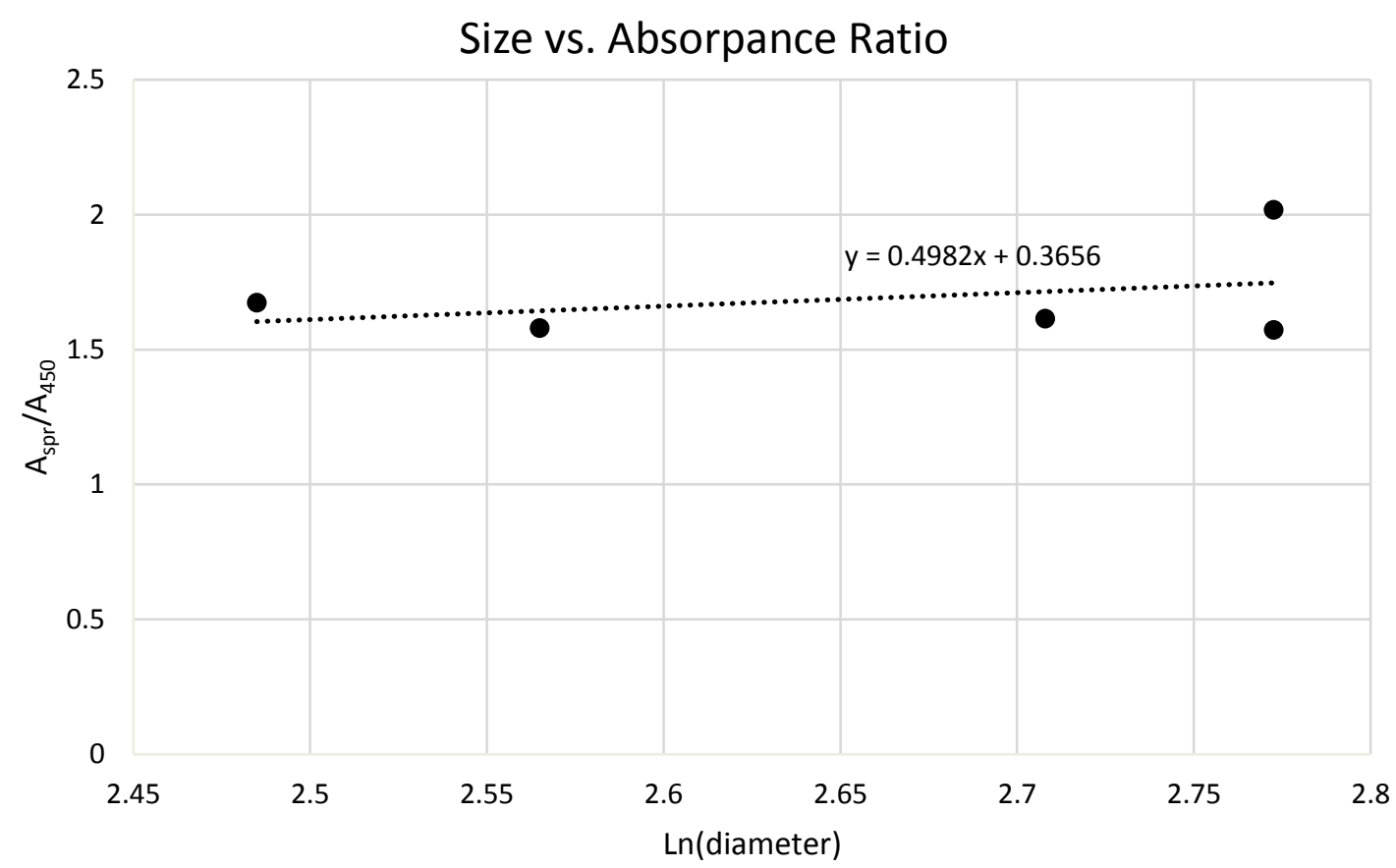

Figure 2: Above are five different GNPs sample. The ratio of the absorption at the SPR to the absorption at 450 nanometers was recorded over the natural log of the diameter of a GNP.

After collecting numerous data points, the function of the fitted line $\left(f\left(x_{i}\right)\right)$, solved through the Method of Least Squares, is represented as:

$$
\hat{y}_{i}=f\left(x_{i}\right)=\hat{b} x_{i}+\hat{b}_{0}
$$


Where the equation fits the experimental set of data in Figure (2). $\hat{b}$ represents the slope of the line. $x_{i}$ represents the natural log value of the diameter of a single gold nanoparticle, and $\widehat{b_{0}}$ represents the ratio of the absorbance of the GNP at the SPR and at 450 nanometers.

After developing the best fit line, $B_{1}$ and $B_{2}$ can be represented as:

$$
\begin{array}{r}
B_{1}=\frac{1}{\widehat{b}} \\
B_{2}=\frac{\widehat{b_{0}}}{\widehat{b}}
\end{array}
$$

Where $B_{1}$ is represented as the reciprocal of the slope of the best-fit line and $B_{2}$ is the ratio of the y-intercept of the best-fit line to the slope.

With these fit parameters, one can now determine the average size of a sample of GNPs with their own experimental values.

\section{DISCUSSION}

By using Beer's Law, the concentration of the solution fell into the range of theoretical values presented in Sigma-Aldrich. The calculated concentration is expected to have some inaccuracy due to the polydispersity of GNPs. The calculated concentration is under the assumption that the GNPs are monodispersed. As presented in the SEM scan in Figure 3, different size GNPs exist in the solution which would cause some discrepancy between the actual and theoretical calculation. Another issue presented is if the reducing agent, trisodium citrate dihydrate, was not fully used up in the reaction. If so, then floating particles of trisodium citrate dihydrate would exist in the 
solution, therefore, Equation (6) would be appropriate to use. By using Equation (6), the amount of error would be reduced since the assumption that two attenuating species exist in solution.
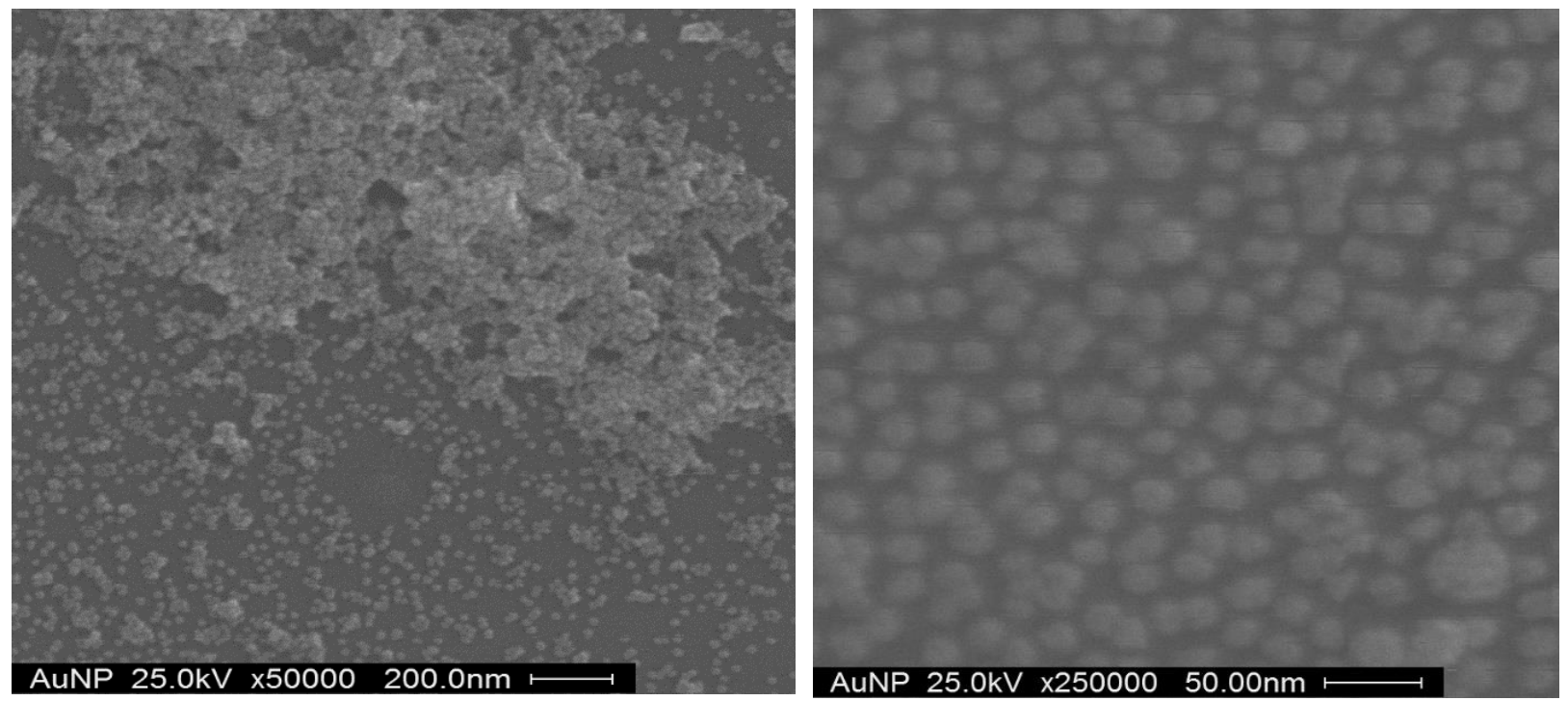

Figure 3: The images are SEM scans of the GNPS at various strengths. The imaged nanoparticles are the same as the ones that were characterized in Figure 1.

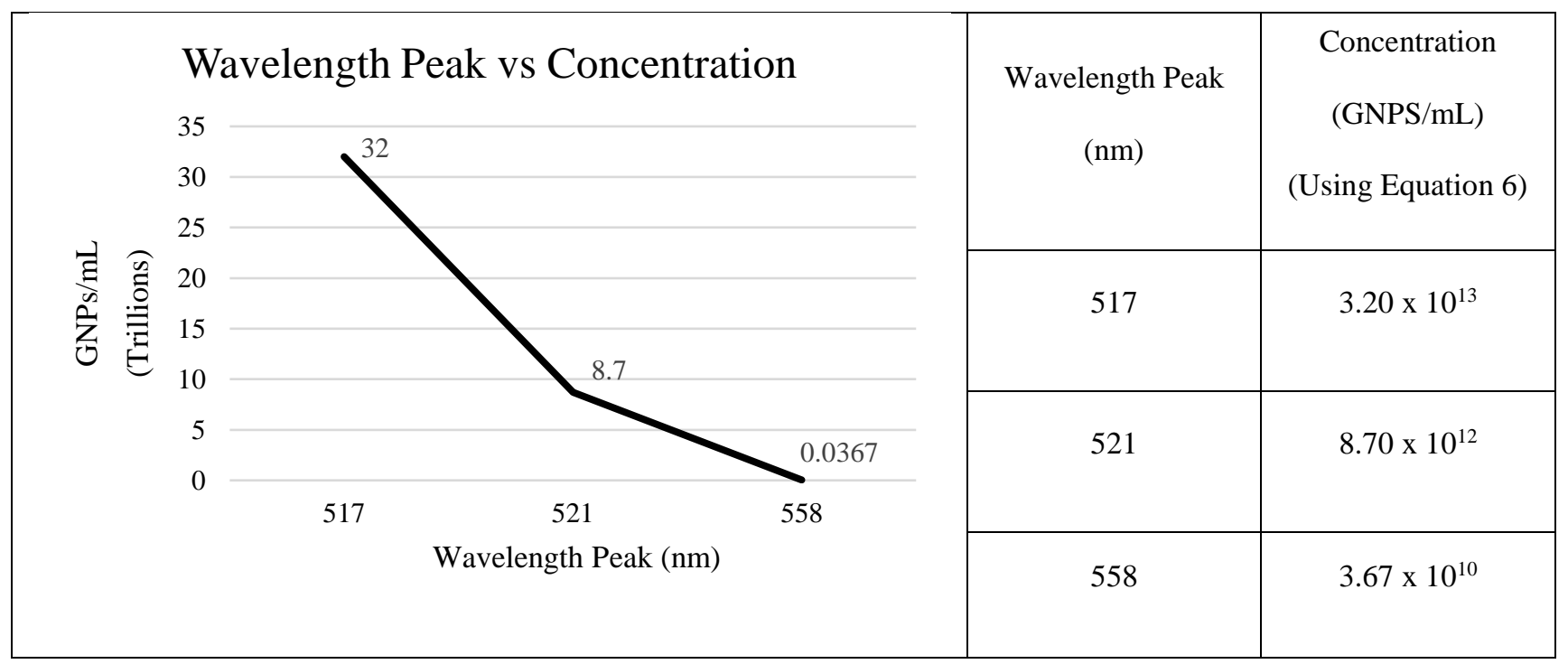

Figure 4: The graph and table present the concentration of GNPs that was solved over various wavelengths recorded through UV-VIS

In Figure 4, a logical correlation between wavelength peak and concentration exists showing validity in using Beer-Lambert's law. As the wavelength of the SPR peak shifts further 
to the right, the larger is the diameter of the GNPs. If the particle size increases, then the concentration of GNP should decrease since the GNPs will be taking up a larger volume of the solution.

By using the data collected from the UV-VIS spectrophotometer in Figure 1, the $\lambda, A_{s p r}$, and $A_{450}$ were calculated. When using Equation (1), both theoretical that were developed in the literature $\left(B_{1}=3.55\right.$ and $\left.B_{2}=3.11\right)$ and experimental values $\left(B_{1}=2.01\right.$ and $\left.B_{2}=3.11\right)$ of $B_{1}$ and $B_{2}$ was used. The diameters that were received were then compared to the average diameter recorded by Sigma Aldrich.

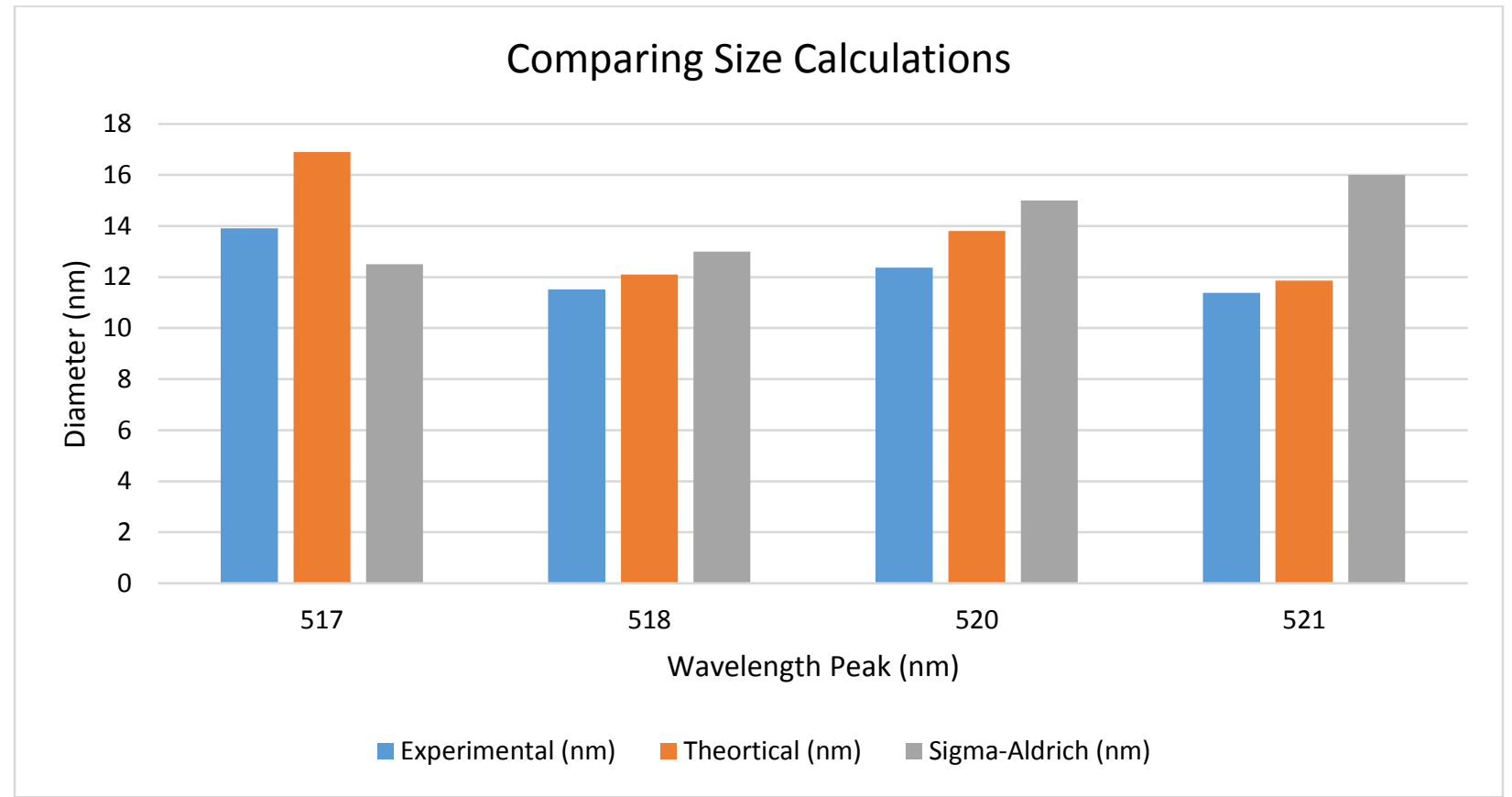




\begin{tabular}{|c|c|c|c|}
\hline \multirow{2}{*}{ Peak (nm) } & $\begin{array}{c}\text { Experimental Fit } \\
\text { Diameter (NM) }\end{array}$ & $\begin{array}{c}\text { Theoretical Fit } \\
\text { Diameter (NM) }\end{array}$ & $\begin{array}{c}\text { Sigma-Aldrich } \\
\text { reported values (NM) }\end{array}$ \\
\hline 517 & 13.9 & 16.9 & $10-15$ \\
\hline 518 & 11.5 & 12.1 & $15-15$ \\
\hline 520 & 12.4 & 13.8 & $15-20$ \\
\hline 521 & 11.4 & 11.9 & \\
\hline
\end{tabular}

Figure 5: The graph and table present the GNP diameter calculated from the experimental fit parameters, theoretical fit parameters, and from Sigma-Aldrich.

\section{CONCLUSION AND RECOMMENDATIONS}

By utilizing Beer-Lambert's law to determine the concentration of GNP in solution a fairly accurate estimate can be drawn if one assumes that no excess trisodium citrate dihydrate remains in the solution and that the size is monodispersed. To improve the accuracy of the data, one should use a DLS instrument to determine the average diameter of the GNP and use the corresponding extinction coefficient. Another improvement that could be made is to filter out any particles of trisodium citrate dihydrate which would allow one to use Equation (4) with high precision.

By using the ratio of the absorbance of the SPR peak to the absorbance at 450 nanometers, a fair approximation of the average diameter of the GNPs suspended in solution can be made. However, higher accuracy may be obtained if one uses their own fit parameters based on their previous GNP samples. By using the equation to determine the size, it cannot be assumed that all the particles are monodispersed. Therefore, the equation does not consider the polydispersity of the GNPs which will result in error. An improvement that can be made is to use 
a wider set of values when plotting the best fit line. Having a larger spread of data points can allow one to use their own experimental values for a wider range of GNP sizes. Using the absorbance peak should not be used as the main determinant of finding the average diameter of the GNPs. The best accuracy may be obtained by using an SEM, TEM, or DLS instrument. 


\section{NOMENCLATURE}

\begin{tabular}{|c|c|c|}
\hline Symbol & Description & Value/Unit \\
\hline$\overline{\epsilon \epsilon}$ & Extinction Coefficient & $\mathrm{M}^{-1} \mathrm{~cm}^{-1}$ \\
\hline$c$ & Concentration & M \\
\hline$f$ & Length of Light Path & $\mathrm{cm}$ \\
\hline$M$ & Atomic Weight of Gold & $197.0 \mathrm{~g}$ \\
\hline$\rho$ & Density of Gold & $19.3 \mathrm{~g} / \mathrm{cm}^{3}$ \\
\hline$d$ & Diameter of GNP & $\mathrm{nm}$ \\
\hline$A$ & Absorbance & - \\
\hline$N$ & Number of Atoms in GNP & - \\
\hline$B_{1}$ & Fit Parameter 1 & - \\
\hline$B_{2}$ & Fit Parameter 2 & - \\
\hline$A_{s p r}$ & Absorbance at SPR peak & - \\
\hline$A_{450}$ & Absorbance at 450 nanometers & - \\
\hline$\hat{B}$ & Slope of Best-Fit Line & - \\
\hline$\widehat{B_{0}}$ & Y-Intercept of Best-Fit Line & - \\
\hline
\end{tabular}

\section{BIBLIOGRAPHY}

Haiss, Wolfgang; Nguyen T. K., Thanh; Aveyard, Jenny; Fernig G., David. "Determination of Size and Concentration of Gold Nanoparticles from UV-Vis Spectra." Analytical Chemistry (2007): 4215-4221. Journal. 
Kumar, Dhiraj; Meenan J., Brian; Mutreja, Isha; D'sa, Raechelle; Dixon Dorian. "Controlling the size and size distribution of gold nanoparticles: A design of experiment study." International Journal of Nanoscience (2012): 125003. Journal.

Lu, Yan; Wang, Lixia; Chen, Dejun; Wang, Gongke. "Determination Of the Concentration and the Average Number of Gold Atoms in a Gold Nanoparticle by Osmotic Pressure." Langmuir (2012): 9282-9287. Journal.

Mie, Gustav. "Beiträge zur Optik trüber Medien, speziell kolloidaler Metallösungen." Annalen der Physik (1908): 377-445.

Sigma-Aldrich. Gold Nanoparticles: Properties and Applications. n.d. 2015.

Swineheart, D. F. "The Beer-Lambert Law." Journal of Chemical Education (1962): 333. Journal.

Turkevich, John, Cooper P Stevenson and James Hillier. "A Study of the Nucleation and Growth Processes in the Synthesis of Colloidal Gold." Discussions of the Faraday Society (1951): $55-75$. 


\section{APPENDIX}

Sigma-Aldrich Reported Values:

\begin{tabular}{|c|c|c|}
\hline Diameter $(\mathrm{nm})$ & $\begin{array}{c}\text { SPR Peak Wavelength } \\
(\mathrm{nm})\end{array}$ & $\begin{array}{c}\text { Extinction Coefficient } \\
\left(\mathrm{M}^{-1} \mathrm{~cm}^{-1}\right)\end{array}$ \\
\hline 5 & $515-520$ & $1.10 \times 10^{7}$ \\
\hline 10 & $515-520$ & $1.01 \times 10^{8}$ \\
\hline 15 & 520 & $3.67 \times 10^{8}$ \\
\hline 20 & 524 & $9.21 \times 10^{8}$ \\
\hline 30 & 526 & $3.36 \times 10^{9}$ \\
\hline 40 & 530 & $1.72 \times 10^{10}$ \\
\hline 50 & 535 & $3.07 \times 10^{10}$ \\
\hline 60 & 540 & $7.70 \times 10^{10}$ \\
\hline 80 & 553 & $1.57 \times 10^{11}$ \\
\hline 100 & 572 & \\
\hline
\end{tabular}

UV-VIS Data:

\begin{tabular}{|c|c|c|}
\hline SPR Peak $(\mathrm{nm})$ & Absorbance at SPR Peak & Absorbance at 450 nm \\
\hline 517 & 1.788574 & 1.069299 \\
\hline 518 & 1.666837 & 1.055632 \\
\hline 520 & 1.830586 & 1.133888 \\
\hline 521 & 1.766482 & 1.123132 \\
\hline
\end{tabular}

